\title{
MHARS: Sistema Móvel de Reconhecimento de Atividades em Ambient Assisted Living
}

\author{
J. D. P. Ribeiro Filho ${ }^{1}$,F. J. da Silva e Silva ${ }^{1}$, L. R. Coutinho ${ }^{1}$, B. de T. P. Gomes ${ }^{1}$ \\ ${ }^{1}$ Programas de Pós-Grad. em Ciência da Computação (PPGCC) e Eng. de Eletricidade (PPGEE) \\ Universidade Federal do Maranhão (UFMA) \\ \{jdanielprf, bertodetacio\}@gmail.com; ffssilva,lrc\}@deinf.ufma.br
}

\begin{abstract}
This paper presents MHARS (Mobile Human Activity Recognition System), a mobile system designed to monitor patients in the context of Ambient Assisted Living (AAL), which allows the recognition of the activities performed by the user, as well as the detection of its intensity in real time. MHARS was designed to be able to gather data from different sensors, to recognize the activities and measure their intensity in different user mobility levels. The system allows the inference of situations regarding the patient health status, and provide the support for executing actions, reacting to events that deserve attention from the patients caregivers. Described experiments demonstrate that MHARS presents good accuracy and has an affordable consumption of mobile resources.
\end{abstract}

Keywords: AAL, Human Activity Recognition, Intensity Measurement, Mobile Computing.

Resumo. O objetivo deste artigo é apresentar o MHARS (Mobile Human Activity Recognition System), um sistema móvel voltado para o acompanhamento pacientes no contexto de Ambient Assisted Living (AAL), que permite o reconhecimento das atividades realizadas pelo usuário bem como a detecção da sua intensidade em tempo real. O MHARS foi projetado para poder obter dados de diferentes sensores, reconhecer as atividades e medir sua intensidade em diferentes niveis de mobilidade do usuário, possui mecanismos para a inferência de situações relativas ao estado de saúde do paciente, bem como o suporte à execução de ações de forma a poder reagir a eventos que mereçam a atenção por parte de seus cuidadores. Experimentos realizados demonstram que o MHARS possui boa acurácia e apresenta um consumo adequado de recursos do dispositivo móvel.

Palavras-chaves: AAL, Reconhecimento de Atividade, Medicação de Intensidade, Computação Móvel.

\section{Introdução}

$\mathrm{Na}$ área da saúde, o termo Ambient Assisted Living (AAL) tem sido empregado para designar um campo de pesquisa multidisciplinar, cujos maiores esforços se concentram no desenvolvimento de sistemas inteligentes de monitoramento das atividades diárias de pacientes (ADL - Activities of Daily Living) vivendo ou transitando em ambientes inteligentes, tais como Smart Homes[Memon et al. 2014]. Sistemas AAL permitem que os interessados em acompanhar mudanças no estado de saúde do paciente (médicos, cuidadores, familiares), possam fazê-lo à certa distância, permitindo que este adquira mais autonomia e mobilidade durante o tratamento. Portanto, sistemas AAL caracterizam uma 
transição do modelo tradicional de atendimento à saúde centrado nas organizações para um modelo no qual predominam serviços individuais de acompanhamento, remoto móvel ou em domicílio, centrados no paciente. Há um amplo espectro de aplicações práticas para sistemas AAL, dentre eles: sistemas de resgate e resposta a emergências, detecção de quedas, sistemas de vigilância por vídeo, etc.

Um dos componentes mais importantes do sistema AAL é o HAR (Human Activity Recognition), cuja tarefa é reconhecer padrões de atividade humana (e.g andar, correr, sentar) a partir de vários tipos de dados do sensores de baixo nível. Dentre a variedade de sensores que podem ser aplicados na detecção de atividades estão os sensores médicos vestíveis (wearable sensors), capazes de coletar dados de sinais fisiológicos (e.g. eletrocardiograma, eletromiografia, frequência cardíaca, volume de oxigênio consumido) ou até mesmo dados que refletem a movimentação corporal (e.g. acelerômetro). Dispositivos pessoais móveis (celulares, tablets e smartphones), em grande parte, também estão equipados com sensores de movimentação ou localização (e.g. acelerômetro, GPS). Sensores ambientais (enviromental sensors) também podem ser utilizados, pois estes coletam informações que ajudam a determinar se as condições ambientais (temperatura, luminosidade, umidade do ar, níveis de gás carbônico) são favoráveis a saúde do paciente. A taxa de acerto no reconhecimento da atividade e sua respectiva intensidade indicam a eficiência do sistema de inferência, e varia de acordo com diversos fatores, dentre eles a acurácia dos dados coletados pelos sensores e técnica de classificação da atividade. A acurácia dos dados coletados depende do tipo e da qualidade do sensor empregado no monitoramento. A fusão ou combinação de dados providos por diferentes sensores ajuda a inferir a situação do paciente, possibilitando uma rápida tomada de decisões e ações, principalmente em situações de emergência.

A detecção da atividade é conveniente em diversos tipos de tratamento de doenças crônicas, principalmente cardíacas (e.g. hipertensão, insuficiência cardíaca, fibrilação atrial) e respiratórias (e.g. bronquite crônica, enfisema pulmonar, asma), pois permite descobrir se o paciente segue ou se distância das rotinas de atividades físicas recomendadas pelos profissionais de saúde. Por exemplo, é possível inferir se o paciente realiza caminhadas ou corridas com frequência ou se este adota uma postura mais sedentária. Em algumas situações é importante verificar como o paciente está reagindo a realização de determinadas atividades físicas, e detectar se o nível de esforço na realização da mesma é compatível com os limites físicos do paciente, impostos pela sua condição crônica, idade, peso e outros fatores. A isso dá-se o nome de medição da intensidade ou do estresse corporal. Em caso de fuga dos padrões de intensidade recomendados, o sistema de detecção da atividade e sua intensidade tomar a decisão de emitir um alerta ao profissional de saúde responsável pela orientação do paciente, ou até solicitar ao paciente que aumente ou diminua o esforço físico durante o treino, a fim de se adequar aos seus limites .

Devido as condições de mobilidade na realização de atividades físicas, alguns sistemas AAL executam o módulo de detecção de atividade e intensidade em dispositivos pessoais móveis, a fim de prover maior flexibilidade na utilização do sistema. Entretanto, dadas as restrições de recursos computacionais em relação ao poder de processamento e consumo de bateria desses dispositivos, é desejável que o referido módulo execute suas tarefas de inferência em tempo hábil e ainda consuma recursos computacionais de maneira sustentável, de modo a oferecer uma relação custo/benefício que indique em sua viabilidade. 
Nesse contexto, o objetivo deste artigo é apresentar a arquitetura, as funcionalidades e os resultados obtidos com a realização de testes preliminares do MHARS (Mobile Human Activity Recognition System), um sistema de reconhecimento de atividades e medição de sua intensidade, voltado para a execução em dispositivos pessoais móveis. Esse sistema está sendo desenvolvido pelo Laboratório de Sistemas Distribuídos (LSD) da UFMA, em regime de colaboração com o Hospital Universitário da referida instituição (HUUFMA), com o objetivo de apoiar o acompanhamento de pacientes portadores de doenças crônicas. MHARS tem como principais características: a capacidade de interagir com diferentes tipos de sensores; reconhecer atividades e medir suas respectivas intensidades em condições de mobilidade; a capacidade de tomar decisões; e o suporte a armazenamento e consulta remota dos dados do paciente.

A estrutura de organização deste artigo é a seguinte. A Seção 2 descreve os componentes e as principais funcionalidades do MHARS. A Seção 3 descreve os primeiros resultados referentes a avaliação do sistema, considerando métricas de verificação da acurácia na detecção da atividade e sua intensidade e ainda métricas de avaliação de desempenho e consumo de recursos. A Seção 4 descreve alguns trabalhos relacionados e promove uma comparação entre eles e o sistema aqui proposto. Por fim, a Seção 5 contém as conclusões deste trabalho e as perspectivas de aplicação do MHARS no cenário da saúde.

\section{MHARS (Mobile Human Activity Recognition System)}

MHARS é um sistema AAL que executa em dispositivos pessoais móveis da plataforma Android, com a finalidade de promover o reconhecimento de padrões de atividades diárias, bem como a medição da intensidade com a qual essas atividades são realizadas, a partir da utilização de sensores. Em relação ao desenvolvimento do sistema, foram estabelecidos os seguintes requisitos: 1) Interação com Sensores: ser capaz de interagir com diversos tipos sensores (vestíveis, portáteis, ou embutidos em ambientes inteligentes), possibilitando a obtenção de diferentes tipos de informações sobre o paciente (e.g. fisiológicos, movimentação corporal e localização) e os ambientes nos quais ele vive (e.g luminosidade, temperatura, qualidade do ar); 2) Reconhecimento de Atividade ser capaz de agrupar, processar e classificar os dados obtidos, de forma a possibilitar o reconhecimento automático das atividades executadas pelo paciente em tempo real; 3) Medição de Intensidade: ser capaz de aferir a intensidade com a qual o paciente monitorado realiza as atividades propostas pelos profissionais de saúde; 4) Inferência de Situação: ser capaz de perceber a situação na qual o paciente se encontra (e.g. estado de normalidade, atenção, urgência ou emergência), por meio da especificação de regras que permitam ao sistema inferir diferentes situações com base na combinação de dados de sensores, da atividade realizada e sua respectiva intensidade, levando em consideração ainda as especificidades do tratamento de cada paciente. 5) Tomada de Decisão: ser capaz de tomar decisões e executar ações adequadas para cada situação em que o paciente pode se encontrar durante a atividade, a fim de garantir a manutenção da saúde. A Figura 1 exibe os componentes do MHARS.

SDAS (Sensor Data Aquisition Service) é o componente responsável pela aquisição dos dados junto aos sensores de baixo nível. Para a obtenção de dados há duas possibilidades. (1) Se o sensor a ser utilizado está embutido no smartphone ou tablet (sensores internos), a aquisição dos dados é feita por meio da API (Application Programing

Interface) de sensores Android. Apenas os sensores requisitados são inicializados, caso estejam disponíveis. (2) Para a aquisição de dados de sensores externos ao dispositivo móvel 


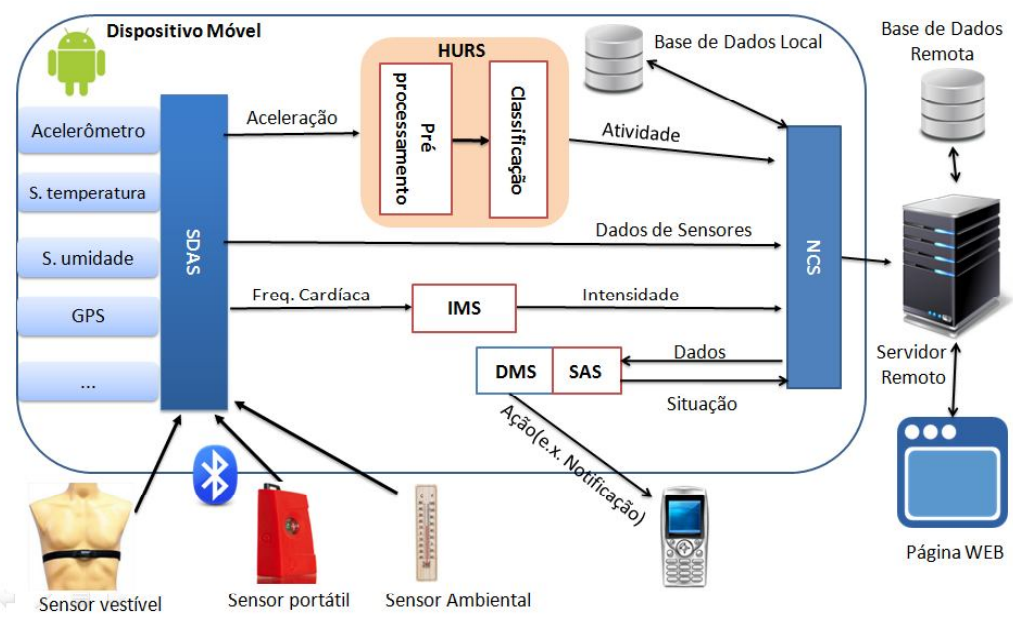

Figura 1. Visão Geral dos Componentes do MHARS

do paciente, utiliza-se de tecnologias de comunicação de curta distância como Bluetooth. Nesse caso, o referido serviço é responsável também pela fase prévia de descoberta e conexão com sensores externos, para posteriormente receber dados desses sensores.

HURS (Human Activity Recognition Service) é o componente responsável pela realização do reconhecimento da atividade realizada pelo paciente. Para realizar esse reconhecimento, o HURS recebe os dados coletados pelo SDAS e realiza uma etapa de pré-processamento, que consiste em realizar a conversão, filtragem e refinamento do sinal do sensor para um formato computacional compreensível ao classificador de dados. Por exemplo, o pré-processamento de sinais obtidos a partir um acelerômetro extrai os ruídos, tendo como resultado um conjunto reduzido de valores numéricos que representam determinadas características relacionadas a aceleração (e.g média, desvio padrão, pico da aceleração, entropia do sinal). $\Lambda$ classificação corresponde a utilização de técnicas de aprendizado de máquina(e.g MLP, árvores de decisão, lógica fuzzy) com a finalidade de reconhecer os padrões de atividade propriamente ditos com base nos valores previamente processados. É necessário que haja um procedimento de treinamento prévio do sistema de reconhecimento. Esse treinamento é feito com base em conjuntos de dados que representam atividades rotuladas (Dataset). O sistema pode utilizar diversos algoritmos de classificação disponibilizados pela biblioteca WEKA [Witten and Frank 2005], que é específica para problemas de reconhecimento de padrões. Esta biblioteca foi incorporada ao HURS. Atualmente esse componente é capaz de reconhecer as seguintes atividades: subindo e descendo escadas, andando, correndo, sentado, em pé e deitado. Para o reconhecimento dessas atividades são empregados os dados obtidos a partir de sensores de aceleração.

IMS (Intensity Measurement Service) é o componente responsável pela medição da intensidade durante a realização de uma atividade, ou seja, mensurar os efeitos fisiológicos (e.g cansaço, estresse, fadiga muscular, produção de ácido láctico) da atividade executada considerando a condição de saúde e os limites físicos de cada paciente. Em geral, o cálculo da intensidade pode considerar diferentes parâmetros, dentre eles a frequência cardíaca ou ECG ou volume de oxigênio consumido. A escolha do melhor parâmetro varia de acordo com os requisitos de monitoramento, tais como os ambientes de monitoramento, bem como a disponibilidade, o grau de intrusividade e a portabilidade dos sensores a serem utilizados. 
A metodologia dominante na literatura relacionada a medição da intensidade das atividades consiste em análises periódicas da frequência cardíaca do paciente [Tanaka et al. 2001]. Quanto mais perto a pessoa estiver da sua frequência cardíaca máxima, maior será a intensidade da atividade. Essa metodologia é a utilizada pelo IMS.

SAS (Situation-Aware Service) é o componente responsável por inferir diferentes situações vivenciadas pelo paciente durante a realização de uma atividade. Em linhas gerais, cada situação é caracterizada pela correlação de um conjunto de dados referentes a atividade em curso, a intensidade de sua realização, e ainda dados sobre a condição crônica e os limites físicos de cada paciente. O SAS tem uma relação intrínseca com o DMS (Decision Making Service), componente responsável pela tomada de decisão e execução de ações diante da ocorrência de determinadas situações. Situações e decisões a serem tomadas, e ações a serem executadas são representadas por meio de uma linguagem de especificação de regras baseadas em XML tipo Evento-Condição-Ação. Profissionais da saúde com auxilio de técnicos podem descrever situações a serem monitoradas por meio de documentos XML. O SAS utilizará as regras descritas nestes documentos para realizar a detecção da situação do paciente. Por exemplo, para pacientes portadores de doenças crônicas cardiovasculares é possível caracterizar uma situação de risco quando esse paciente executa, durante um longo período de tempo, uma atividade física cujo tipo ou nível de intensidade mensurado não seja compatível com as recomendações médicas. Nessa situação, a decisão a ser tomada pode ser alertar o paciente sobre o risco da referida atividade e/ou comunicar o médico responsável via SMS, para que este tome conhecimento da situação do paciente.

NCS (Network Connections Service) é componente responsável pela conexão oportunista com redes 3G/4G ou WI-FI, possibilitando o envio de dados a um servidor remoto tão logo exista um rede disponível. Enquanto a conexão com a Internet não esteja disponível, os dados são armazenados em uma base local. O servidor remoto contém uma base de dados relacionadosz ao monitoramento do paciente. Essa base pode armazenar

dados de baixo nível, a fim de construir um histórico de medições dos sensores utilizados pelo paciente em atividade ao longo do tempo de acompanhamento, e/ou informações de mais alto nível, tais como aquelas que representam o histórico de situações vivenciadas pelo paciente durante o tratamento, ou registros de decisões e ações que foram tomadas em caso e situações de emergência. A base de dados sobre o paciente pode ser consultada remotamente por profissionais da saúde por meio de um plataforma Web.

\section{Avaliação}

Para a avaliação preliminar do MHARS foi adotada uma metodologia de avaliação baseada em estudos de caso, que consistiu em experimentos envolvendo o acompanhamento de 10 pessoas durante a realização das seguintes atividades: subindo e descendo escadas, andando, correndo, sentado, em pé e deitado. A definição das atividades foi realizada em conjunto com profissionais da área da saúde do HUUFMA. O objetivo principal da avaliação é analisar a taxa de acerto, ou seja, a capacidade do sistema em reconhecer diferentes atividades (estáticas ou dinâmicas) utilizando sensores de aceleração em diferentes partes do corpo (cintura, coxa, peito). O objetivo secundário da avaliação é analisar se o volume de recursos (processador e memória) exigidos para a execução do sistema é favorável a sua utilização em dispositivos móveis de uso convencional dos pacientes. 
Para a execução dos experimentos foram utilizados um Smartphone Motorola Moto G II com o sistema operacional Android 4.4.2 KitKat ${ }^{1}$ e o dispositivo vestível Zephyr BioHarness $3^{2}$. Para o reconhecimento das atividades foram utilizados dois acelerômetros: um embutido no smartphone (sensor interno), e o outro embutido no dispositivo vestível (sensor externo). Cada paciente utilizou o smartphone em duas posições diferentes (coxa e cintura). O dispositivo vestível foi projetado para ser utilizado apenas no peito. O objetivo era analisar a variação na taxa de acerto em relação a diferentes posições do acelerômetro. Ambos os sensores foram configurados para operar em uma frequência de $50 \mathrm{~Hz}$, visto que na literatura este valor é utilizado de forma recorrente, sendo considerado bastante adequado para a detecção tanto de atividades estacionárias (e.g. parado) como de atividades dinâmicas (e.g. correndo). Além disso, essa frequência é considerada como aceitável em relação ao consumo de bateria dos dispositivos móveis. Para a medição da intensidade da atividade, foi utilizado apenas o sensor de frequência cardíaca embutido no dispositivo vestível, operando a uma frequência de $1 \mathrm{~Hz}$. Essa frequência é padrão do dispositivo e para o modelo adotado não pode ser configurado.

O método de pré-processamento utilizado nos experimentos consistiu na obtenção dos seguintes valores representativos: a média e o desvio padrão dos valores de cada eixo $(\mathrm{X}, \mathrm{Y}, \mathrm{Z})$, bem como a raiz quadrada da média obtida a partir das médias referentes a cada eixo. Esses valores são repassados ao classificador para que execute o reconhecimento da atividade. É importante frisar que outros métodos de pré-processamento poderiam ser adotados, entretanto, outras possibilidades requerem maior consumo de recursos computacionais, de forma que não são considerados adequados para o reconhecimento de atividades em tempo real a partir de dispositivos pessoais móveis, devido a limitações na capacidade de processamento e memória.

Nesta avaliação, os processos de reconhecimento da atividade e sua intensidade foram executados em intervalos de 2,58 segundos. Isso quer dizer que, considerando uma frequência de $50 \mathrm{~Hz}$, os processos de pré-processamento, classificação e medição da intensidade são executados com base na quantidade de 128 amostras de dados de aceleração a cada ciclo de reconhecimento. Essa janela de tempo e respetiva quantidade de amostras são consideradas suficientes para a detecção de atividades que são executadas repetidamente. Por exemplo, uma pessoa caminhando normalmente realiza entre 90 e 130 passadas por minuto, o que corresponde a respectivamente entre 1,5 e 2,7 passos por segundo. Sendo assim, uma janela de tempo de 2,58 segundos é mais do que suficiente para reconhecer uma atividade de caminhada[Anguita et al. 2013].

O classificador de dados precisa ser treinado a partir da análise de dados representativos de atividades rotuladas, a fim de que posteriormente esse componente consiga reconhecer corretamente as atividades. Convém explicar que o procedimento prévio de treinamento exige a execução de processos computacionais intensivos, e que por esse motivo não são realizados no dispositivo móvel, mas sim no servidor remoto. Para a construção do DataSet foi adotada a seguinte estratégia: do total de amostras coletadas, $70 \%$ foi destinado ao treinamento do classificador, enquanto que os $30 \%$ restantes foram destinados a sua validação, ou seja, avaliar a taxa de acerto do classificador em relação ao reconhecimento da atividade após o treinamento. A divisão do DataSet foi necessária para

\footnotetext{
${ }^{1}$ http://www.lojaoficialmotorola.com.br/novo-moto-g

${ }^{2}$ http: / / zephyranywhere.com/products/bioharness-3
} 
verificar a presença de overfitting [Russell and Norvig 2003], um problema caracterizado quando o classificador não consegue reconhecer uma determinada atividade para a qual foi treinado com base na análise de amostras desconhecidas. Isso indica que o classificador, mesmo treinado, não consegue generalizar o reconhecimento da atividade, ou seja, ele está viciado.

Diversas técnicas de aprendizagem de máquina são empregadas para o reconhecimento de atividades humanas, entre as quais se destacam as árvores de decisão. Este trabalho investiga a utilização do um algoritmo de árvore de decisão chamado Random Forest para o reconhecimento de atividades humanas. Para isso, este trabalho treinou e avaliou o algoritmo selecionado para a classificação das diversas atividades representadas no Dataset. Como trabalho futuro pretende-se realizar uma comparação da taxa de acerto do Random Forest com outros algoritmos de aprendizagem de máquina.

O Random Forest é capaz de reconhecer a atividade executada com base na utilização de múltiplas árvores de decisão[Witten and Frank 2005]. Uma árvore de decisão é composta por nodos que representam testes/decisões a serem tomadas com base em valores de entrada (dados pré- processados). Durante o processo de decisão, a árvore é percorrida de cima para baixo, começando pelo nodo raiz da árvore até alcançar a folha que contém a atividade compatível com os valores de entrada. Árvores de decisão são aplicadas em diversos trabalhos que envolvem o reconhecimento de padrões, tanto para aplicações gerais, como especificamente no campo de reconhecimento de atividades humanas [Hoseinitabatabaei et al. 2013].

Para a medição do tempo de reconhecimento da atividade foram obtidos o timestamp de início da fase de pré-processamento e o de término do processo de classificação. O tempo de reconhecimento equivale à diferença entre esses tempos. Para a medição do volume de recursos computacionais exigidos para a execução do sistema foi utilizada a ferramenta $\mathrm{DDMS}^{3}$, que faz parte do SDK Android. O sistema foi executado durante uma hora, sendo que foram realizadas medições em intervalos de 6 minutos, totalizando 10 medições. $\mathrm{O}$ valor final que representa o consumo de recursos é obtido a partir da média dos valores obtidos em relação ao uso de CPU e memória em cada medição.

\subsection{Resultados e Discussões}

A tabela 1 contém os resultados referentes à taxa de acerto obtida, considerando os diferentes tipos de atividades realizadas e as variadas posições dos acelerômetros.

Em relação ao tipo de atividade realizada, é possível observar, de acordo com os resultados apresentados nos experimentos, que as atividades estacionárias (baixa variação de aceleração) apresentam maiores taxas de acerto, quando comparadas com as atividades dinâmicas (alta variação de aceleração). Esses resultados são atribuídos ao fato do reconhecimento de atividades dinâmicas requerer sensores precisos em relação a detecção de variações na aceleração em curtos espaços de tempo. Dependendo da precisão do sensor, os dados fornecidos para o treinamento do algoritmo de classificação podem não ser os mais representativos para a atividade realizada, a ponto de serem confundidos com dados de outra atividade. A calibração dos sensores também tende a interferir no grau de precisão dos dados coletados, afetando o treinamento do algoritmo de classificação e, consequentemente, resultar na diminuição na taxa de acerto no reconhecimento da atividade.

\footnotetext{
${ }^{3}$ http://developer.android.com/tools/debugging/ddms.html
} 
Tabela 1. Taxa de Acertos da Modelo de Classificação de Atividades

\begin{tabular}{|c|c|c|c|c|}
\hline Atividade & Tipo & \multicolumn{3}{|c|}{ Posição Acelerômetro } \\
\hline & & Coxa & Peito & Cintura \\
\hline Correndo & Dinâmica & $82,2 \%$ & $63,4 \%$ & $88,2 \%$ \\
\hline Subindo Escada & Dinâmica & $67,1 \%$ & $46,4 \%$ & $61,4 \%$ \\
\hline Descendo Escada & Dinâmica & $69,2 \%$ & $57,6 \%$ & $74,9 \%$ \\
\hline Andando & Dinâmica & $75,4 \%$ & $71,6 \%$ & $89,3 \%$ \\
\hline Em pé & Estacionária & $91,2 \%$ & $63,8 \%$ & $87,2 \%$ \\
\hline Sentado & Estacionária & $90,1 \%$ & $41,4 \%$ & $88,3 \%$ \\
\hline Deitado & Estacionária & $88,2 \%$ & $72,2 \%$ & $85,4 \%$ \\
\hline \multicolumn{2}{|c|}{ Total } & $80,48 \%$ & $59,47 \%$ & $82,05 \%$ \\
\hline
\end{tabular}

Em relação a posição dos acelerômetros, de acordo com os resultados apresentados nos experimentos, é possível observar que as taxas de acerto são maiores quando o acelerômetro está localizado na cintura e na coxa, quando comparados aos resultados obtidos na posição do peito. Esses resultados são atribuídos ao fato de que, quando posicionados na cintura ou na coxa, os acelerômetros conseguem detectar a movimentação do usuário com maior precisão, dado que essas partes do corpo sofrem maior impacto e agitação durante a realização da atividade que o peito.

Analisando os resultados obtidos de forma geral, considera-se que o sistema de reconhecimento da atividade possui taxa de acerto satisfatória (82\%), indicando que o mesmo possui confiabilidade suficiente para ser utilizado no acompanhamento de pacientes. Isso é reforçado por dados encontrados na literatura, onde afirma-se que um sistema é capaz de detectar de maneira satisfatória atividades humanas caso possua uma taxa de acerto maior que 70\% [Hoseinitabatabaei et al. 2013]. Acredita-se que no futuro poderemos obter taxas de acerto ainda maiores, usando abordagens como: a utilização de sensores mais precisos, o uso de um maior volume de amostras para treinamento do algoritmo de classificação, a análise sistemática do comportamento de diversos algoritmos constantes da biblioteca WEKA e a utilização de classificadores construídos através de uma combinação destes algoritmos. É importante reforçar que o principal foco desta avaliação preliminar era avaliar a capacidade de reconhecer diferentes atividades considerando variadas posições dos sensores de aacelereção no corpo do usuário.

Em relação ao tempo médio gasto para o reconhecimento das atividades no dispositivo móvel, este é de aproximadamente 750 milissegundos, com poucas variações. Isso se deve ao fato de que estes experimentos utilizaram uma única técnica de classificação, de forma que a complexidade em termos de tempo para executar a busca em uma árvore binária é de nlogn. Esses resultados permitem afirmar que o tempo gasto para o reconhecimento da atividade é razoável para a utilização do sistema para o acompanhamento de pacientes em tempo real, visto que o tempo de resposta em relação ao reconhecimento da atividade é baixo. Em relação ao consumo de CPU e memória, os valores médios obtidos foram respectivamente $45 \%$ e $30 \mathrm{MB}$. Esses valores são considerados aceitáveis para a utilização de sistemas de reconhecimento de atividades em móveis populares, cujas configurações se aproximam a do smartphone utilizado no experimento. 


\section{Trabalhos Relacionados}

Esta seção descreve resumidamente outros trabalhos relacionados ao reconhecimento de atividades humanas e medição de sua respectiva intensidade. Há trabalhos que realizam apenas o reconhecimento de atividade, entretanto, para diminuir o escopo das comparações, eles não serão considerados. A comparação entre este trabalho e os relacionados é feita com base nos seguintes critérios: I) sensores utilizados; II) capacidade de reconhecimento da atividade e medição da intensidade em condições de mobilidade; III) capacidade de inferir situações do paciente e tomar decisões; e IV) capacidade de armazenamento e consulta remota dos dados do paciente.

O artigo [Carvalho et al. 2011] apresenta um sistema para o acompanhamento de pacientes em ambiente domiciliar, promove o reconhecimento de atividades e intensidade (repouso, comendo, caminhando, fazendo atividade doméstica, indo dormir, acordando) com base na utilização de acelerômetro (afixado na cintura do paciente nos experimentos de avaliação). Esse sistema também tem a capacidade de tomar decisões de acordo com a situação do paciente, bem como promove o armazenamento dos dados do paciente em um servidor remoto. Em [Tapia et al. 2007], assim como o MHARS, também utiliza acelerômetro e frequência cardíaca, tanto para o reconhecimento da atividade como para a medição da intensidade em tempo real, considerando 14 atividades diferentes. Entretanto, diferentemente do MHARS, os trabalhos [Carvalho et al. 2011] e [Tapia et al. 2007] não executam o processo de reconhecimento da atividade ou sua intensidade em dispositivos móveis. Esses trabalhos ao contrário do MHARS, não suportam o acompanhamento do paciente em cenários de mobilidade (e.x. caminhando na rua ou correndo na praia ou em um parque). Eles também não apresentam em sua avaliação a taxa de acerto obtida no reconhecimento das atividades propostas.

O sistema proposto em [Eid et al. 2013] é voltado para ser utilizado no acompanhamento de atletas durante a execução de atividades esportivas, mas sem o objetivo de diferenciá-las. O objetivo do sistema é apenas a medição da intensidade da atividade realizada. Neste trabalho, a medição da intensidade é realizada com base na utilização de um sensor de frequência cardíaca, utilizando para isso um microprocessador Arduíno ${ }^{4}$, suportando, portanto, cenário de mobilidade restrito ao ambiente desportivo do atleta. Esse sistema, assim como o MHARS, tem a capacidade de inferir situações e tomar decisões. Entretanto, diferente do MHARS, essa tomada de decisão é feita apenas com base na intensidade, pois a atividade não é reconhecida.

\section{Conclusões}

Este trabalho apresentou o MHARS, um sistema AAL móvel com foco no acompanhamento de pacientes, que permite tanto o reconhecimento de atividades, como a detecção da intensidade na qual estas estão sendo realizadas. Foram apresentados os principais componentes do sistema, suas funcionalidades e aspectos de implementação. O sistema foi avaliado considerando a capacidade do mesmo em reconhecer diferentes atividades (estacionárias e dinâmicas)a partir do uso de sensores de aceleração em variadas posições. Os resultados dessas avaliações indicam que o sistema alcançou uma taxa de acerto aceitável. Os resultados da avaliação do tempo de reconhecimento das atividades indicaram que o sistema é capaz de determinar a atividade realizada em pouco tempo, de forma que o

\footnotetext{
${ }^{4}$ http: / / www . arduino.cc/
} 
mesmo pode ser utilizado para o acompanhamento de pacientes em tempo real. O volume de recursos computacionais exigidos para a execução do sistema também foi mensurado. Os resultados dessa avaliação indicaram que o sistema possui viabilidade para ser executado em dispositivo pessoais móveis de uso comum, que possuem restrições em relação a suas capacidades de memória e processamento, quando comparado a computadores pessoais de maior porte (PCs e notebooks). Foi realizada também a comparação do sistema proposto com alguns trabalhos relacionados recentes. De forma geral, o MHARS é mais abrangente em relação às capacidades utilizadas como critérios de comparação, possibilitando tanto o reconhecimento da atividade como de sua intensidade, suportando cenários de pacientes com mobilidade, promovendo o acesso remoto as informações coletadas e ainda oferecendo suporte a tomada de decisões e ações diante de diferentes situações.

\section{Agradecimentos}

Os autores gostariam de agradecer a FAPEMA (Fundação de Amparo à Pesquisa e Desenvolvimento Científico do Maranhão) pelo apoio a este trabalho.

\section{Referências}

Anguita, D., Ghio, A., Oneto, L., Parra, X., and Reyes-Ortiz, J. L. (2013). A public domain dataset for human activity recognition using smartphones. In European Symposium on Artificial Neural Networks, Computational Intelligence and Machine Learning, ESANN.

Carvalho, S. T., Copetti, A., and Loques, O. (2011). Sistema de computação ubíqua na assistência domiciliar à saúde. Journal of Health Informatics, 3:51-57.

Eid, M., Saad, U., and Afzal, U. (2013). A real time vibrotactile biofeedback system for optimizing athlete training. In Haptic Audio Visual Environments and Games (HAVE), 2013 IEEE International Symposium on, pages 1-6.

Hoseinitabatabaei, S. A., Gluhak, A., and Tafazolli, R. (2013). A survey on smartphonebased systems for opportunistic user context recognition. ACM Comput. Surv., 45(3):27:1-27:51.

Memon, M., Wagner, S. R., Pedersen, C. F., Beevi, F. H. A., and Hansen, F. O. (2014). Ambient assisted living healthcare frameworks, platforms, standards, and quality attributes. Sensors, 14(3):4312-4341.

Russell, S. J. and Norvig, P. (2003). Artificial Intelligence: A Modern Approach. Pearson Education, 2 edition.

Tanaka, H., Monahan, K. D., and Seals, D. R. (2001). Age-predicted maximal heart rate revisited. Journal of the American College of Cardiology, 37(1):153-156.

Tapia, E., Intille, S., Haskell, W., Larson, K., Wright, J., King, A., and Friedman, R. (2007). Real-time recognition of physical activities and their intensities using wireless accelerometers and a heart rate monitor. In Wearable Computers, 2007 11th IEEE International Symposium on, pages 37-40.

Witten, I. H. and Frank, E. (2005). Data Mining: Practical Machine Learning Tools and Techniques, Second Edition (Morgan Kaufmann Series in Data Management Systems). Morgan Kaufmann Publishers Inc., San Francisco, CA, USA. 